\title{
Rapid Formation of Gas Giant Planets around M Dwarf Stars
}

\author{
Alan P. Boss \\ Department of Terrestrial Magnetism, Carnegie Institution of Washington, 5241 Broad \\ Branch Road, NW, Washington, DC 20015-1305 \\ boss@dtm.ciw.edu
}

\begin{abstract}
Extrasolar planet surveys have begun to detect gas giant planets in orbit around $\mathrm{M}$ dwarf stars. While the frequency of gas giant planets around $\mathrm{M}$ dwarfs so far appears to be lower than that around $G$ dwarfs, it is clearly not zero. Previous work has shown that the core accretion mechanism does not seem to be able to form gas giant planets around $\mathrm{M}$ dwarfs, because the time required for core formation scales with the orbital period, which lengthens for lower mass stars, resulting in failed (gas-poor) cores unless the gaseous protoplanetary disk survives for $>10$ Myr. Disk instability, on the other hand, is rapid enough $\left(\sim 10^{3}\right.$ yrs) that it should be able to form gas giant protoplanets around even low mass stars well before the gaseous disk disappears. A new suite of three dimensional radiative, gravitational hydrodynamical models is presented that calculates the evolution of initially marginally gravitationally unstable disks with masses of 0.021 to $0.065 M_{\odot}$ orbiting around stars with masses of 0.1 and $0.5 M_{\odot}$, respectively. The models show that gas giant planets are indeed likely to form by the disk instability mechanism in orbit around $\mathrm{M}$ dwarf stars, the opposite of the prediction for formation by the core accretion mechanism. This difference offers another observational test for discriminating between these two theoretical end members for giant planet formation. Ongoing and future extrasolar planet searches around $\mathrm{M}$ dwarfs by spectroscopy, microlensing, photometry, and astrometry offer the opportunity to help decide between the dominance of the two mechanisms.
\end{abstract}

Subject headings: stars: planetary systems - stars: low-mass, brown dwarfs

\section{Introduction}

M dwarf stars dominate the nearby stellar population: within $10 \mathrm{pc}$ of the sun, there are least 236 M dwarfs, but only 21 known G dwarfs (Henry et al. 1997). In spite of this fact, 
spectroscopic extrasolar planet surveys have concentrated on G dwarfs, in hopes of finding Solar System analogues. Gravitational microlensing surveys at first set only upper bounds $(<45 \%)$ on the frequency of multiple-Jupiter-mass planets orbiting between $1 \mathrm{AU}$ and 7 AU around M dwarf stars toward the Galactic bulge (Gaudi et al. 2002), but recently the first microlensing detections of Jupiter-mass companions orbiting $M$ dwarfs appear to have been accomplished (Bond et al. 2004; Udalski et al. 2005), and more should be on the way. Spectroscopic planet surveys have begun to focus more on $\mathrm{M}$ dwarfs, though a few $\mathrm{M}$ dwarfs have been included for several years. One of these, GJ 876, has a pair of gas giant planets (Marcy et al. 1998, 2001) as well as an even lower mass planet of uncertain composition (Rivera et al. 2005). Butler et al. (2004) and Bonfils et al. (2005a) have found evidence for giant planets of Neptune-mass orbiting two more M dwarfs, GJ 436 and Gl 581. There are hints from other spectroscopic surveys as well that $M$ dwarfs may have a significant frequency $(\leq 13 \%)$ of short period giant planets (Endl et al. 2003).

Given that $\mathrm{M}$ dwarfs have masses as low as $\sim 0.1$ that of $\mathrm{G}$ dwarfs, their most massive planets might be expected to be similarly lower in mass and therefore harder to detect than those orbiting $\mathrm{G}$ dwarfs, whose planets range up to masses of $\sim 10 M_{J}$ (Jupiter masses). GJ 876 was the first $M$ dwarf found spectroscopically to have planets, implying that its planetary spectroscopic signatures are relatively large. Hence, GJ 876's gas giants may be indicative of the most massive planets to be found around M dwarfs, at least on relatively short period orbits. GJ 876's two gas giant planets have minimum masses of $\sim 2.1 M_{J}$ and $\sim 0.56 M_{J}$ (Rivera et al. 2005), consistent with this argument, given GJ 876's mass of $0.32 M_{\odot}$. If the range of gas giant masses is shifted downward by a factor of $\sim 3$ or more for $\mathrm{M}$ dwarfs compared to $\mathrm{G}$ dwarfs, it will take considerably more effort to obtain as complete a census of $\mathrm{M}$ dwarf planets compared to $\mathrm{G}$ dwarf planets, even though the lower mass of the star itself works in favor of detection by either spectroscopy or astrometry. The intrinsic faintness of $\mathrm{M}$ dwarfs also adds to the observational burden, particularly for spectroscopic surveys, which are photon-limited in their precision.

Young $\mathrm{M}$ dwarf stars appear to have dust disks similar to those around T Tauri stars (Kalas, Liu, \& Matthews 2004; Liu et al. 2004; Liu 2004; Mohanty, Jayawardhana, \& Basri 2005), so there is no a priori observational reason to believe that $M$ dwarfs should not be able to form planets in much the same manner as their somewhat more massive siblings.

Relatively little theoretical work has been done on the formation of planets around low mass stars, because of the general obsession with explaining the origin of our own planetary system. Wetherill (1996), however, found that Earth-like planets were just as likely to form from the collisional accumulation of planetesimals around $\mathrm{M}$ dwarfs with half the mass of the Sun as they were to form around G dwarfs. Boss (1995) studied the thermodynamics of 
protoplanetary disks around stars with masses from 1.0 to 0.1 solar mass, and found that the location of the ice condensation point only moved inward by a few AU at most when the stellar mass was decreased through this range. In the core accretion model of giant planet formation (Mizuno 1980), this result implied that gas giant planets should be able to form equally well around $\mathrm{M}$ dwarfs, though perhaps at somewhat smaller orbital distances.

Core accretion, however, is a process once thought to have required times of order 10 Myr to form a core massive enough to accrete a gaseous envelope orbiting a G dwarf star (Pollack et al. 1996). Theorists have worked hard to shorten the core accretion time scale to as little as $\sim 4 \mathrm{Myr}$ at 5.2 $\mathrm{AU}$ (Inaba, Wetherill, \& Ikoma 2003), given the evidence for typical disk lifetimes of $\sim 3$ Myr (Haisch, Lada, \& Lada 2001; Eisner \& Carpenter 2003) or less in regions of high mass star formation (Bally et al. 1998; Bricẽno et al. 2001). The time scale for core growth can be shortened to $\sim 1$ to $2 \mathrm{Myr}$ by assuming that inward Type-I migration hastens the accumulation of solid cores (Alibert et al. 2005), but Type-I migration appears to be more of a fatal danger to core accretion in the presence of gas than an aid to gas giant planet formation (Kominami, Tanaka, \& Ida 2005): in spite of efforts to lengthen them, Type-I migration time scale estimates remain considerably shorter than core growth time scales and gaseous disk lifetimes, and considerably shorter than was assumed to be the case by Alibert et al. (2005).

In a turbulent, magnetorotationally unstable disk, Type-I migration can become at least in part a stochastic process, resulting in a component of random walk in semimajor axis and hence a distribution of time scales for inward migration (Laughlin, Steinacker, \& Adams 2004; Nelson 2005), possibly allowing some cores to accrete gaseous envelopes before migrating inward. However, such turbulence is limited to ionized regions of the disk, whereas the planet-forming midplane from $\sim 1 \mathrm{AU}$ to $\sim 10 \mathrm{AU}$ is likely be magnetically dead (e.g., Gammie 1996), leaving Type-I migration as a significant danger for core accretion.

Core accretion is expected to take even longer to produce a gas giant planet around an $\mathrm{M}$ dwarf, as the lower stellar mass leads to longer orbital periods at a given distance from the star. Core accretion thus appears to be too slow to produce Jupiter-mass planets in orbit around M dwarfs before the disk gas disappears (Laughlin et al. 2004). Laughlin et al. (2004) found that core accretion required significantly more than $\sim 10 \mathrm{Myr}$ to form a gas giant in orbit around a $0.4 M_{\odot}$ star, compared to a similar calculation where a gas giant formed in $\sim 3 \mathrm{Myr}$ around a $1 M_{\odot}$ star. Roberge et al. (2005) found no evidence for significant gas in the debris disk of the M1 star AU Microscopii, with an age of $\sim 12 \mathrm{Myr}$. Laughlin et al. (2004) concluded that gas giant planets should be rare around M dwarfs, but that failed cores (i.e., roughly Neptune-mass cores that grew too slowly to accrete a significant gaseous envelope) should be common. 
Metallicities are notoriously difficult to measure in $M$ dwarfs, but a recent study of wide binary systems including M dwarf secondaries by Bonfils et al. (2005b) has permitted a photometric calibration for $\mathrm{M}$ dwarf metallicities, assuming that the $\mathrm{M}$ dwarf secondaries have the same metallicities as their F, G, or K primaries. Bonfils et al. (2005b) thereby determined that the metallicities of two of the M dwarfs (Gl 876 and Gl 436) known from Doppler spectroscopy to have giant planets are very close to solar. They also found that the metallicities of 47 nearby $\mathrm{M}$ dwarfs are slightly lower on average than those of nearby $\mathrm{F}, \mathrm{G}$, or $\mathrm{K}$ dwarfs, consistent with the $\mathrm{M}$ dwarfs being somewhat older on average. These results suggest that $\mathrm{M}$ dwarfs are able to form giant planets even with solar metallicities, though the sample size obviously needs to be increased greatly.

In this paper we examine the possibility of forming gas giant planets around $\mathrm{M}$ dwarfs by the competing mechanism of disk instability (Cameron 1978; Boss 1997, 1998, 2003, 2004, 2005; Mayer et al. 2002, 2004). In the disk instability mechanism, a marginally gravitationally unstable protoplanetary disk forms spiral arms that can lead to the formation of gravitationally-bound clumps of Jupiter-mass on a time scale of $\sim 10$ orbital periods, typically $\sim 10^{3}$ yrs or less for a protoplanetary disk at orbital distances of $\sim 10$ AU. Disk instability represents a potentially rapid mechanism for gas giant planet formation around $\mathrm{M}$ dwarfs, if $\mathrm{M}$ dwarfs have suitable marginally gravitationally unstable disks.

\section{Numerical Methods}

The calculations were performed with a finite volume code that solves the three dimensional equations of hydrodynamics and radiative transfer, as well as the Poisson equation for the gravitational potential. The code is second-order-accurate in both space and time (Boss \& Myhill 1992) and has been used extensively in previous disk instability studies (e.g., Boss 2003, 2004, 2005).

The equations are solved on spherical coordinate grids with $N_{r}=101, N_{\theta}=23$ in $\pi / 2 \geq \theta \geq 0$, and $N_{\phi}=256$ or 512 . The radial grid extends from 4 AU to 20 AU with a uniform spacing of $\Delta r=0.16 \mathrm{AU}$. The $\theta$ grid is compressed toward the midplane in order to ensure adequate vertical resolution $\left(\Delta \theta=0.3^{\circ}\right.$ at the midplane). The $\phi$ grid is uniformly spaced to prevent any azimuthal bias. The central protostar wobbles in response to the growth of disk nonaxisymmetry, preserving the location of the center of mass of the star and disk system. The number of terms in the spherical harmonic expansion for the gravitational potential of the disk is $N_{Y l m}=32$ or 48 . The Jeans length criterion is monitored throughout the calculations to ensure proper spatial resolution: the numerical grid spacings in all three coordinate directions should remain less than $1 / 4$ of the local Jeans length in order to avoid 
the possibility of spurious fragmentation caused by poor spatial resolution.

The boundary conditions are chosen at both 4 AU and 20 AU to absorb radial velocity perturbations. Mass and linear or angular momentum entering the innermost shell of cells at $4 \mathrm{AU}$ is added to the central protostar and thereby removed from the hydrodynamical grid. During a typical model, several Jupiter-masses of disk gas are accreted by the central protostar, yielding an average mass accretion rate of $\sim 10^{-6}$ to $\sim 10^{-5} \mathrm{M}_{\odot} / \mathrm{yr}$. A much smaller amount of mass and momentum reaches the outermost shell of cells at $20 \mathrm{AU}$ and is effectively removed from the calculation: the disk mass piles up in this shell and is assigned zero radial velocity. The inner and outer boundary conditions are designed to absorb incident mass and momentum, rather than to reflect mass and momentum back into the main grid.

As in Boss $(2003,2004)$, the models treat radiative transfer in the diffusion approximation, with no radiative losses or gains occurring in regions where the vertical optical depth $\tau$ drops below 10. In very low density regions interior to the initial disk structure, the disk temperature is assumed to be the same as that of the initial disk at that radial location. Above the disk, the temperature is set equal to the envelope temperature (Table 1). The envelope temperatures of $30 \mathrm{~K}$ to $50 \mathrm{~K}$ are conservatively high estimates for $\mathrm{M}$ dwarf protostars, given that temperatures of $\sim 50 \mathrm{~K}$ appear to be appropriate for solar-mass protostars (Chick \& Cassen 1997).

\section{Initial Conditions}

The models calculate the evolution of a 0.1 or $0.5 M_{\odot}$ central protostar surrounded by a protoplanetary disk with a mass $\left(M_{d}\right)$ ranging from 0.021 to $0.065 M_{\odot}$, respectively, between $4 \mathrm{AU}$ and $20 \mathrm{AU}$ (Table 1). The initial protoplanetary disk structure is based on the following approximate vertical density distribution (Boss 1993) for an adiabatic, self-gravitating disk of arbitrary thickness in near-Keplerian rotation about a point mass $M_{s}$

$$
\begin{gathered}
\rho(R, Z)^{\gamma-1}=\rho_{o}(R)^{\gamma-1} \\
-\left(\frac{\gamma-1}{\gamma}\right)\left[\left(\frac{2 \pi G \sigma(R)}{K}\right) Z+\frac{G M_{s}}{K}\left(\frac{1}{R}-\frac{1}{\left(R^{2}+Z^{2}\right)^{1 / 2}}\right)\right]
\end{gathered}
$$

where $R$ and $Z$ are cylindrical coordinates, $\rho_{o}(R)$ is a specified midplane density, and $\sigma(R)$ is a specified surface density. The disk surface occurs where $\rho(R, Z)=0$. The adiabatic pressure (used only for defining the initial model - the radiative transfer solution includes a full thermodynamical treatment) is defined by $p=K \rho^{\gamma}$, where $K$ is the adiabatic constant and $\gamma$ is the adiabatic exponent. The adiabatic constant is $K=1.7 \times 10^{17}$ (cgs units) and 
$\gamma=5 / 3$ for the initial model. The radial variation of the midplane density is chosen to be a power law that ensures near-Keplerian rotation throughout the disk

$$
\rho_{o}(R)=\rho_{o 4}\left(\frac{R_{4}}{R}\right)^{3 / 2}
$$

where $\rho_{\text {o4 }}$ varies between $1.3 \times 10^{-11} \mathrm{~g} \mathrm{~cm}^{-3}$ and $6.0 \times 10^{-11} \mathrm{~g} \mathrm{~cm}^{-3}$ (Table 1) and $R_{4}=4$ AU. The resulting disk surface density falls off roughly as $\sigma \propto r^{-1}$ over most of the disk, falling to $\sigma \propto r^{-3 / 2}$ near the outer edge. A low density halo $\rho_{h}$ of gas and dust infalls onto the disk, with

$$
\rho_{h}(r)=\rho_{h 4}\left(\frac{R_{4}}{r}\right)^{3 / 2}
$$

where $\rho_{h 4}=1.0 \times 10^{-14} \mathrm{~g} \mathrm{~cm}^{-3}$ and $r$ is the spherical coordinate radius.

The initial velocity field vanishes inside the disk, except for Keplerian rotation, while in the halo the initial velocity field is given (based on conservation of energy) by

$$
\begin{gathered}
v_{r}=-\left(\frac{G M_{s}}{r}\right)^{1 / 2} \cos \theta \\
v_{\theta}=\left(\frac{G M_{s}}{r}\right)^{1 / 2} \sin \theta \\
v_{\phi}=\left(\frac{G M_{s}}{r}\right)^{1 / 2} .
\end{gathered}
$$

The translational $\left(v_{r}, v_{\theta}\right)$ velocity field in the halo is simply vertical infall toward the disk midplane, and the azimuthal velocity is taken to be Keplerian. The chosen velocity field is an analytical approximation that is convenient to implement and that retains the essence of more exact solutions (e.g., Cassen \& Moosman 1981).

The initial disk temperatures are based on the models of Boss (1995), with midplane temperatures of $300 \mathrm{~K}$ at $4 \mathrm{AU}$, decreasing monotonically outward to a distance of $\sim 6.7$ $\mathrm{AU}$, where they are assumed to become uniform at an outer disk temperature of $T_{o}$. $T_{o}$ is varied in order to make sure that the outer disk is marginally gravitationally unstable in terms of the gravitational stability parameter $Q$, i.e., initial minimum values of $Q \sim 1.5$ are assumed (Table 1). In low optical depth regions such as the disk envelope, the temperature is assumed to be equal to a fixed value, $T_{e}$, with $T_{e}$ varied between $30 \mathrm{~K}$ and $50 \mathrm{~K}$, and chosen 
to be similar to $T_{o}$. The Rosseland mean opacities used in the radiative transfer solution have been updated to include the dust grain opacities calculated by Pollack et al. (1994).

\section{Results}

Table 1 lists the initial conditions for the models with $N_{\phi}=256$. The models with $N_{\phi}=512$ were started at a point in the evolution of the $N_{\phi}=256$ models when the disks were forming spiral arms and clumps dense enough that the Jeans conditions were on the verge of being violated. At that time, the $N_{\phi}=256$ models were doubled in azimuthal resolution to provide starting conditions for the $N_{\phi}=512$ models.

Figure 1 depicts the initial surface density profile for model $5 \mathrm{C}$, showing that the inner regions begin from a state of gravitational stability, while the outer regions are closer to marginal gravitational stability $\left(Q_{\min }=1.50\right.$ for model $\left.5 \mathrm{C}\right)$. The increased gravitational stability inside $\sim 6 \mathrm{AU}$ is a direct result of the assumed higher initial disk temperatures inside this radius (Boss 1995). Figure 1 also illustrates that the assumed initial disk model does not result in a single power law for $\sigma(r)$, but rather a smooth falloff with radius toward $\sigma \propto r^{-3 / 2}$ near the 20 AU outer boundary.

Figure 2 shows that model $5 \mathrm{CH}$ has formed a number of clearly defined clumps after just 208 years of evolution around a protostar with a mass of $0.5 M_{\odot}$. Clump formation occurs soon after the initial disk develops spiral arms that transport angular momentum outward and mass inward, resulting in a significant depletion of the gas from the innermost disk (Figure 3). This occurs in spite of the inner region being gravitationally stable gravitational torques from one-armed spirals propagating at $\sim 8 \mathrm{AU}$ and beyond are able to drive disk gas inward and onto the central protostar. Several Jupiter masses of gas are accreted from the inner disk by the central protostar.

Strong spiral arms begin forming first in the innermost, unstable region of the disk, i.e., around $\sim 6 \mathrm{AU}$, because of the combination of relatively low $Q$ and short orbital periods there. Spiral arms form at greater distances as the evolution proceeds. The densest clumps tend to form at distances of $\sim 8 \mathrm{AU}$ for the same reasons, which are ultimately tied to the initial temperature profiles in the disk midplanes (Figure 1; Boss 1995).

The densest clump seen in Figure 2 has a mass of $0.93 M_{J}$ within 0.01 of the maximum clump density of $8.8 \times 10^{-10} \mathrm{~g} \mathrm{~cm}^{-3}$ (Figure 4). This mass is larger than the Jeans mass at

the average density $\left(2.3 \times 10^{-10} \mathrm{~g} \mathrm{~cm}^{-3}\right)$ and temperature $(69 \mathrm{~K}$; Figure 5$)$ of the clump of $0.69 M_{J}$, implying that the clump is gravitationally bound in the absence of shear. Note that while the average clump temperature is $69 \mathrm{~K}$, peak clump temperatures are $\sim 100 \mathrm{~K}$, double 
the minimum outer disk temperature $\left(T_{o}\right)$ in this model of $50 \mathrm{~K}$ - the highest temperatures tend to occur near the edges of the clump where the disk gas is being compressionally heated. The ratio of thermal to gravitational energy for this clump is 0.80 , also implying that it is gravitationally bound.

The effective spherical radius of the densest clump in Figure 2 is 0.82 AU, which is somewhat larger than the critical radius for tidal stability (Boss 1998) for this clump of 0.68 AU. However, given that the clump is banana-shaped at this point in time, with its minimum extent laying along the critical radial direction, the clump should be capable of contracting to smaller sizes and higher densities and should thus survive, though perhaps not before having lost some mass through tidal forces. Mass is also likely to be gained by accretion from the disk once the clump becomes a well-defined protoplanet, as was found in the virtual protoplanet models of Boss (2005). The clump has a nearly circular orbit at this point, with an eccentricity of 0.0005 and semimajor axis of $7.7 \mathrm{AU}$. If the clump survives to form a protoplanet and undergoes substantial inward migration to a stable orbit, its final orbital eccentricity is likely to be unrelated to this initial eccentricity, but will depend on the mechanism responsible for the inward migration.

Figure 6 illustrates that the densest clump seen in Figure 2 is well-resolved with respect to the Jeans criteria. Hence, this clump, as well as the somewhat less dense clumps seen in Figure 2, do not appear to be spurious results. Once the clumps form, they contract to higher densities and may then begin to violate the Jeans criterion for the radial coordinate, the most restrictive Jeans criterion at the orbital distance of the clumps (Figure 6). However, given that the clumps formed without violating the Jeans criteria, their subsequent contraction to higher densities must be viewed as physically realistic, though any indication of subfragmentation during such a poorly-resolved phase would be suspicious and is in fact not seen. The clumps attain masses of $\sim 1.1$ to $1.3 M_{J}$ within 10 yrs after the phase shown in Figure 2, with effective spherical radii less than or comparable to their critical tidal radii. Their orbital eccentricities increase to $\sim 0.1$ during this time interval.

The clumps seen in model $5 \mathrm{CH}$ in Figure 2 eventually are torn apart by tidal forces as the present grid-based code is unable to provide the locally higher spatial resolution needed for the clumps to continue their contraction to ever higher and higher densities. The models of Boss (2005) showed that clumps become better defined and longer-lived as the spatial resolution is increased, but the question of clump survival and evolution will ultimately require treatment by an adaptive mesh refinement (AMR) code. Smoothed-particle-hydrodynamics (SPH) models of disk instabilities with locally defined smoothing lengths (Mayer et al. 2002, 2004) have already demonstrated the need for such locally enhanced spatial resolution. Even though the clumps seen in Figure 2 are doomed to disappear eventually by the nature of the 
present calculations, new clumps continue to form and evolve, implying that the disks are determined to eventually form self-gravitating clumps.

All of the other models of disks orbiting $0.5 M_{\odot}$ stars (models $\mathrm{A}, \mathrm{AH}, \mathrm{B}, \mathrm{BH}$, and D) behave in the same manner as models $\mathrm{C}$ and $\mathrm{CH}$ : the disks develop strong spiral arms that interact and form self-gravitating clumps with masses ranging from $\sim 0.3$ to $1.9 M_{J}$. These masses appear to be roughly consistent with the minimum masses of the gas giant planets orbiting the M dwarf GJ 876 (Marcy et al. 1998, 2001).

We now turn to the models with disks orbiting $0.1 M_{\odot}$ stars (models $1 \mathrm{~A}, 1 \mathrm{~B}, 1 \mathrm{C}$, and 1D). Figure 7 shows the outcome of 446 yrs of evolution of model 1C, which had a disk with an initial mass of $0.021 M_{\odot}$. Even with these low values of the stellar and disk masses, model $1 \mathrm{C}$ managed to form a self-gravitating clump in a very short period of time.

The densest clump seen in Figure 7 at 4 o'clock for model $1 \mathrm{C}$ has a mass of $1.1 M_{J}$ within 0.05 of the maximum clump density of $1.6 \times 10^{-10} \mathrm{~g} \mathrm{~cm}^{-3}$. This mass is larger than the Jeans mass at the average density $\left(2.2 \times 10^{-11} \mathrm{~g} \mathrm{~cm}^{-3}\right)$ and temperature (39 K; Figure 8) of the clump of $0.96 M_{J}$, implying that the clump once again is gravitationally bound. Figure 8 again shows that the highest temperatures do not necessarily occur at the location of the density maxima, but rather near the clump edges. The clump has an orbital eccentricity of 0.12 and a semimajor axis of $7.4 \mathrm{AU}$ at this time.

Models 1A and 1B also formed gravitationally bound clumps. Model 1D formed clumps, but the densest clump analyzed did not appear to be quite dense enough to be gravitationally bound, at least for the limited number of time steps when the calculational data was saved for later analysis. Periodic clump formation persisted throughout the 1300 yrs that model 1D was evolved, so there is no clear evidence that the disk instability process could not result in the formation of planetary-mass companions to even lower mass objects, i.e., brown dwarfs, provided that these objects have marginally gravitationally unstable disks at some phase of their evolution.

Movies of models $1 \mathrm{~A}, 1 \mathrm{~B}, 1 \mathrm{C}, 1 \mathrm{D}, 5 \mathrm{~A}, 5 \mathrm{AH}, 5 \mathrm{~B}$, and $5 \mathrm{BH}$ in mpeg format may be downloaded from the following web site: http://www.dtm.ciw.edu/boss/ftp/mpeg/mdwarf/.

\section{Conclusions}

These models suggest that there is no reason why $M$ dwarf stars should not be able to form gas giant protoplanets rapidly, if a disk instability can occur. This prediction differs fundamentally from that of the core accretion mechanism, which does not appear to be 
able to form gas giant planets around $\mathrm{M}$ dwarf on time scales shorter than typical disk lifetimes (Lauglin et al. 2004). Disk instability may even be able to form planetary-mass objects in orbit around brown dwarfs with suitably unstable disks, though this has not been demonstrated by the present set of models.

Ongoing and future extrasolar planet searches will answer the question of whether or not gas giant planets form frequently around low mass stars. If $\mathrm{M}$ dwarfs turn out not to have very many gas giant planets, this could be interpreted as either a failure of disk instability to produce gas giant planets, or as a failure of $\mathrm{M}$ dwarfs to have disks massive enough to be marginally gravitationally unstable. If $\mathrm{M}$ dwarfs do turn out to have a significant frequency of gas giant planets, this would imply that disk instability is responsible for their formation, given the apparent inability of core accretion to form gas giants fast enough (Laughlin et al. 2004).

Regardless of the situation for the gas giant planets, the Neptune-mass planets that have been found around $\mathrm{M}$ dwarfs are unlikely to have formed by disk instability, unless massive gaseous envelopes can be removed by stellar heating and irradiation, a process that may be reasonably efficient for solar-mass stars (Vidal-Madjar et al. 2004; Hebrard et al. 2005). Rather, such planets are likely to have formed by the same collisional accumulation process that led to the terrestrial planets in our Solar System. Such a finding would not preclude the existence of gas giant planets orbiting at greater distances from $\mathrm{M}$ dwarfs, as is the case for GJ 876, planets which presumably formed by disk instability.

Given the extreme imbalance in the numbers of the closest stars of different spectral types, $\mathrm{M}$ dwarfs are a natural choice for astrometric planet searches, where closeness is a primary virtue. The low mass of the primary helps as well to enable astrometric detections of even lower mass planets. Ground-based efforts are underway at Carnegie's Las Campanas Observatory to detect brown dwarf and gas giant planet companions to M, L, and T dwarfs by astrometry (Boss et al. 2006). Space-based astrometric searches by NASA's Space Interferometry Mission (SIM) should lower the detectable planet mass to Earth-masses or below around M dwarfs. Space-based transit missions (COROT, Kepler) will be able to detect many giant planets orbiting $\mathrm{M}$ dwarfs, as well as the ongoing ground-based microlensing and spectroscopic searches. We can expect that all of these efforts will combine to determine the gas giant planet frequency around $\mathrm{M}$ dwarfs, and thereby help to determine the most likely mechanism for the formation of their giant planets.

I thank Fred Adams for his valuable comments on the manuscipt and Sandy Keiser for her critical computer systems expertise. This research was supported in part by NASA Planetary Geology and Geophysics grant NNG05GH30G and by NASA Astrobiology Institute grant NCC2-1056. The calculations were performed on the Carnegie Alpha Cluster, the 
purchase of which was partially supported by NSF Major Research Instrumentation grant MRI-9976645. 


\section{REFERENCES}

Alibert, Y., et al. 2005, A\&A, 434, 343

Bally, J., et al. 1998, AJ, 116, 854

Bond, I. A., et al. 2004, ApJ, 606, L155

Bonfils, X., et al. 2005a, A\&A, 443, L15

- 2005b, A\&A, 442, 635

Boss, A. P. 1993, ApJ, 417, 351

—. 1995, Science, 267, 360

—. 1997, Science, 276, 1836

—. 1998, ApJ, 503, 923

—. 2003, ApJ, 599, 577

—. 2004, ApJ, 610, 456

—. 2005, ApJ, 629, 535

Boss, A. P., \& Myhill, E. A. 1992, ApJS, 83, 311

Boss, A. P., et al. 2006, PASP, in preparation

Briceño, C., et al. 2001, Science, 291, 93

Butler, R. P., et al. 2004, ApJ, 617, 580

Cameron, A. G. W. 1978, Moon Planets, 18, 5

Cassen, P., \& Moosman, A. 1981, Icarus, 48, 353

Chick, K. M., \& Cassen, P. 1997, ApJ, 477, 398

Eisner, J. A., \& Carpenter, J. M. 2003, ApJ, 598, 1341

Endl, M., et al. 2003, AJ, 126, 3099

Gammie, C. F. 1996, ApJ, 553, 174

Gaudi, B. S., et al. 2002, ApJ, 566, 463

Haisch, K. E., Lada, E. A., \& Lada, C. J. 2001, ApJ, 553, L153

Hebrard, G., et al. 2005, in Extrasolar Planets: Today and Tomorrow, ASP Conference Series 321, p. 203

Henry, T. J., et al. 1997, AJ, 114, 388

Inaba, S. , Wetherill, G. W., \& Ikoma, M. 2003, Icarus, 166, 46 
Kalas, P., Liu, M. C., \& Matthews, B. C. 2004, Science, 303, 1990

Kominami, J., Tanaka, H., \& Ida, S. 2005, Icarus, 178, 540

Laughlin, G., Bodenheimer, P., \& Adams, F. C. 2004, ApJ, 612, L73

Laughlin, G., Steinacker, A., \& Adams, F. C. 2004, ApJ, 608, 489

Liu, M. C. 2004, Science, 305, 1442

Liu, M. C., et al. 2004, ApJ, 608, 526

Marcy, G. W., et al. 1998, ApJ, 505, L147

—. 2001, ApJ, 556, 296

Mayer, L., Quinn, T., Wadsley, J., \& Stadel, J. 2002, Science, 298, 1756

—. 2004, ApJ, 609, 1045

Mizuno, H. 1980, Prog. Theor. Phys., 64, 544

Mohanty, S., Jayawardhana, R., \& Basri, G. 2005, ApJ, 626, 498

Nelson, R. P. 2005, A\&A, 443, 1067

Rivera, E., et al. 2005, ApJ, 634, 625

Roberge, A., et al. 2005, ApJ, 626, L105

Pollack, J. B., et al. 1994, ApJ, 421, 615

—. 1996, Icarus, 124, 62

Udalski, A., et al. 2005, ApJ, 628, L109

Vidal-Madjar, A., et al. 2004, ApJ, 604, L69

Wetherill, G. W. 1996, Icarus, 119, 219 


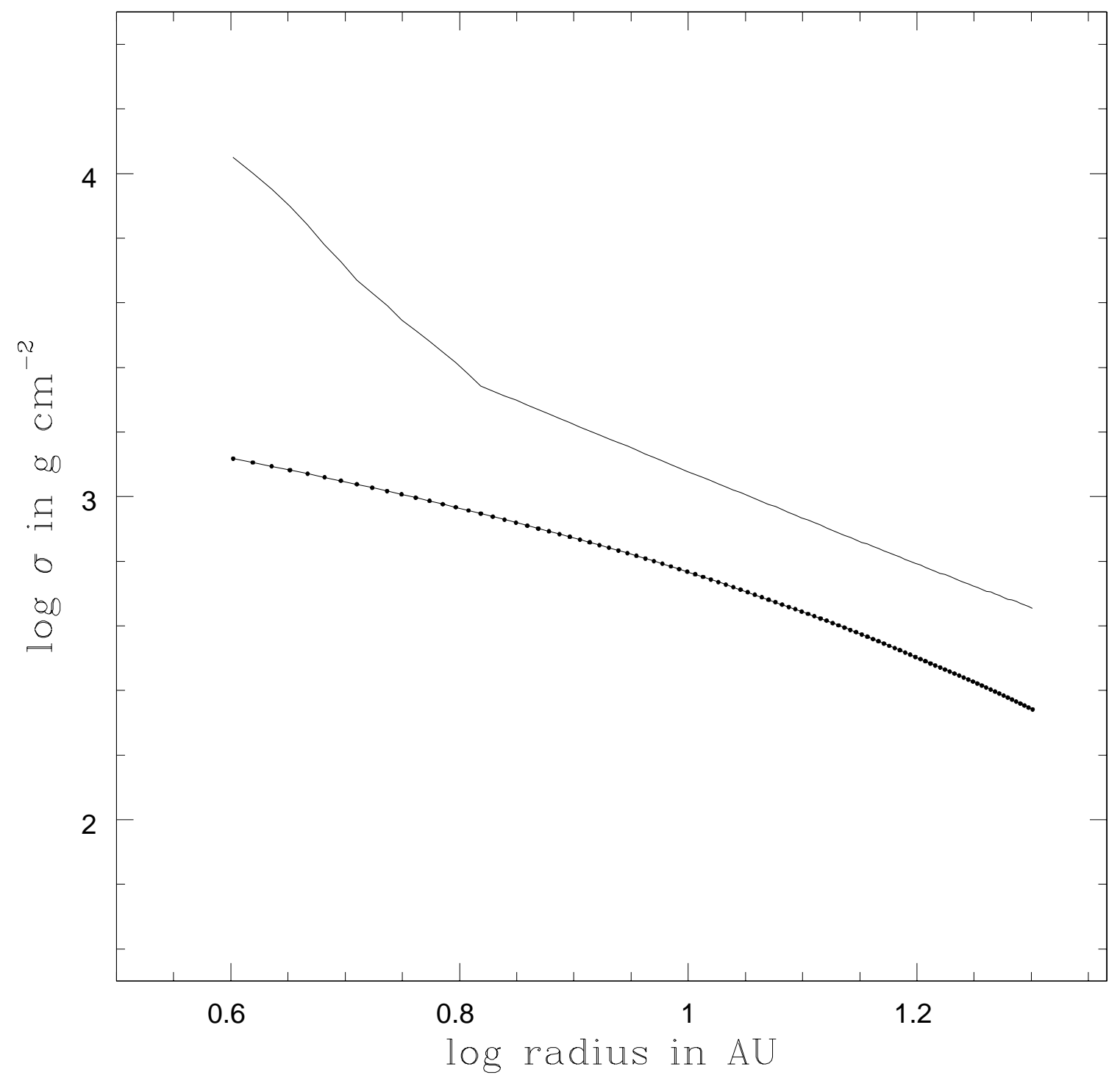

Fig. 1. - Radial (azimuthally averaged) profile of the disk gas surface density (dots) in the initial model 5C, compared to the surface density needed for $Q=1$ (solid line). The initial disk surface density is too low by about a factor of two for the outer disk to have $Q=1$. 


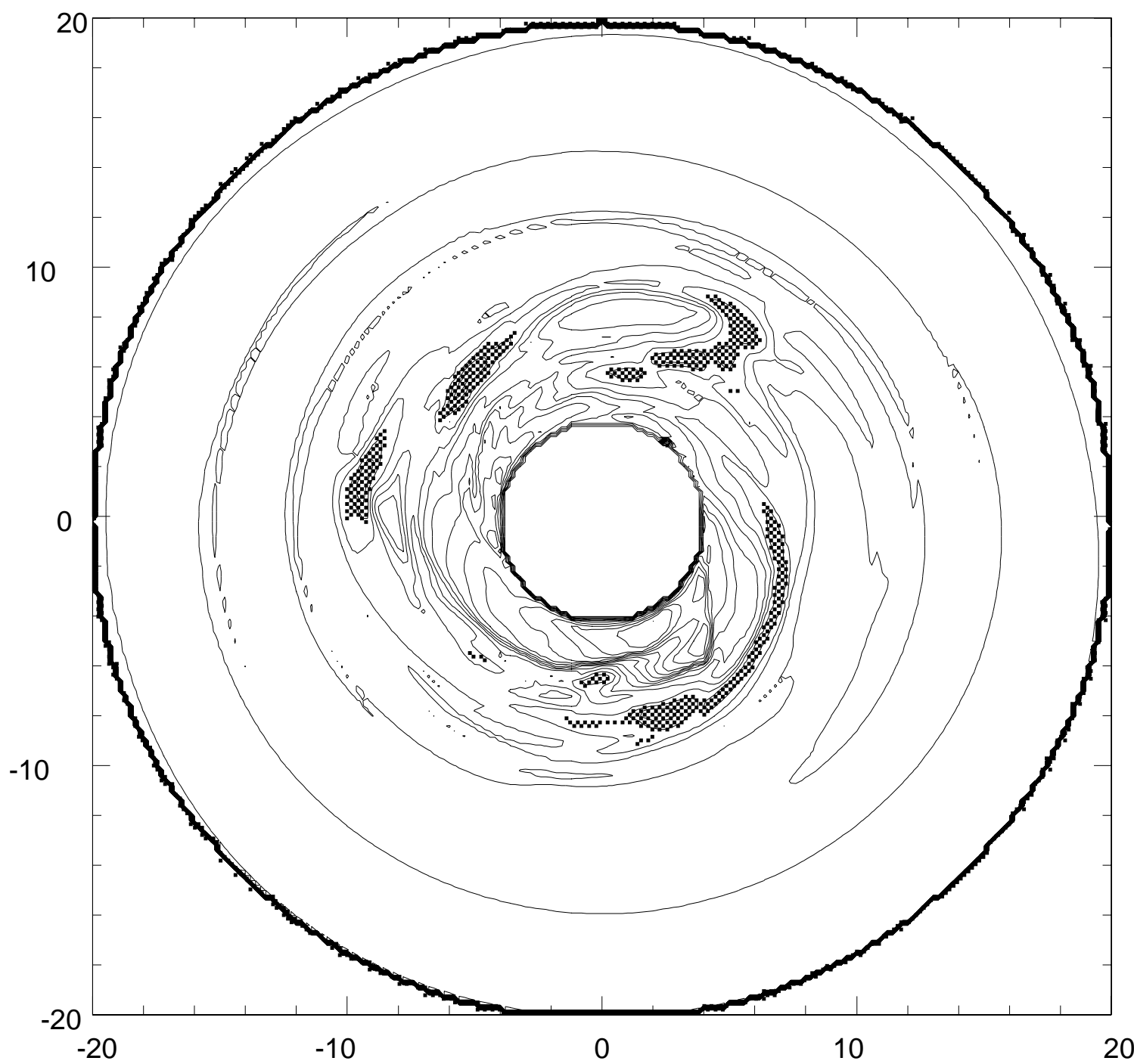

Fig. 2.- Equatorial density contours for model $5 \mathrm{CH}$ after 208 yrs of evolution. The entire disk is shown, with an outer radius of $20 \mathrm{AU}$ and an inner radius of $4 \mathrm{AU}$, through which mass accretes onto the central protostar. Hashed regions denote spiral arms and clumps with densities higher than $10^{-10} \mathrm{~g} \mathrm{~cm}^{-3}$. Density contours represent factors of two change in density. 


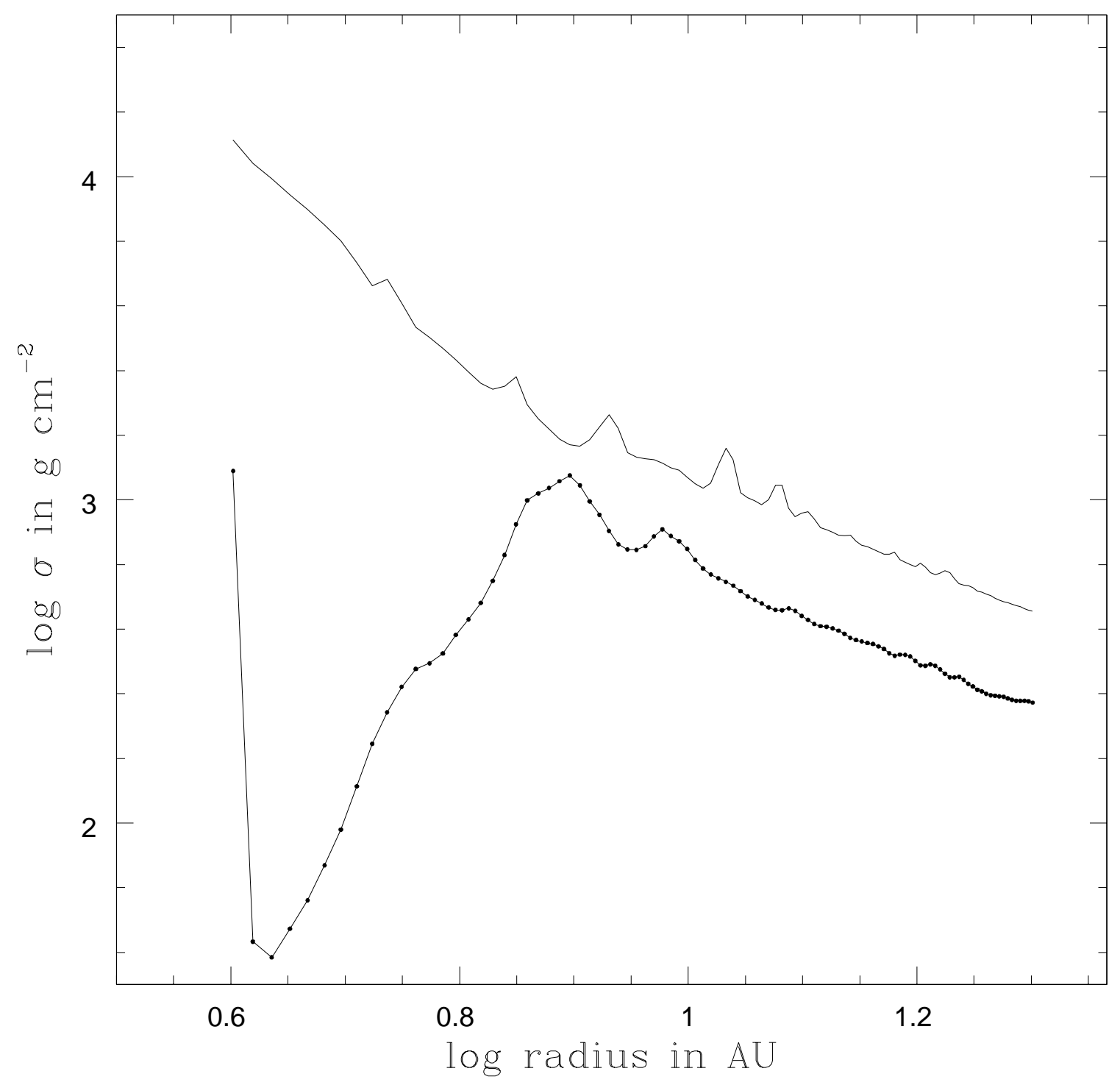

Fig. 3.- Radial (azimuthally averaged) profile of the disk gas surface density (dots) for model $5 \mathrm{CH}$ after 208 yrs of evolution, compared to the surface density needed for $Q=1$ (solid line). The innermost disk gas (several Jupiter-masses) has largely accreted onto the central protostar. 


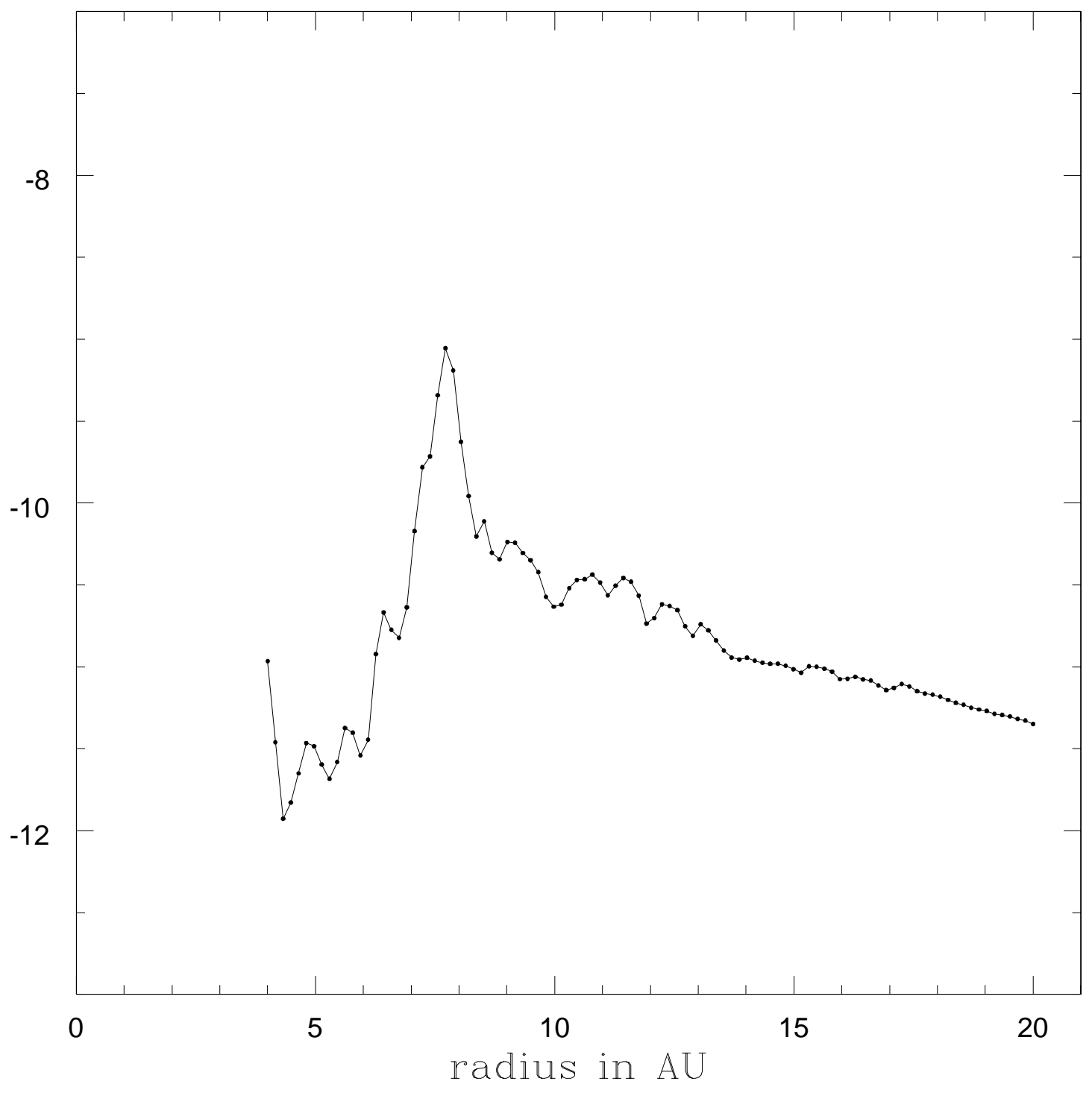

Fig. 4.- Radial density profile ( $\log$ of the density in $\mathrm{g} \mathrm{cm}^{-3}$ ) passing through the densest clump (with a mass of $\sim 0.93 M_{J}$ ) seen in Fig. 2 at 9 o'clock for model $5 \mathrm{CH}$. 


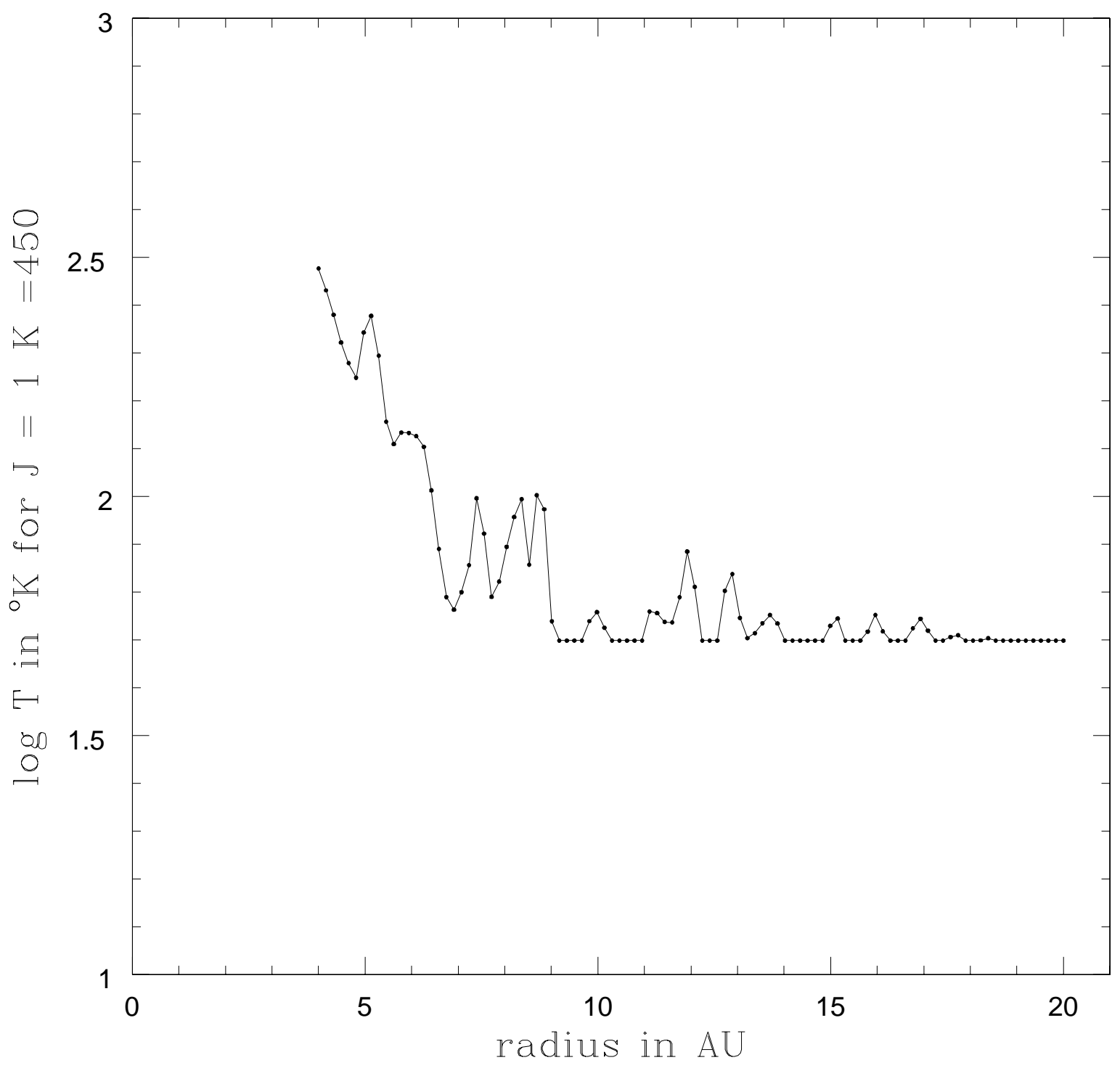

Fig. 5.- Radial temperature profile for model $5 \mathrm{CH}$, as in Fig. 4, taken along a ray through the densest clump in Fig. 2. Temperatures in the outer disk are not allowed to drop below their initial values, which accounts for the thermal floor evident outside 9 AU. 


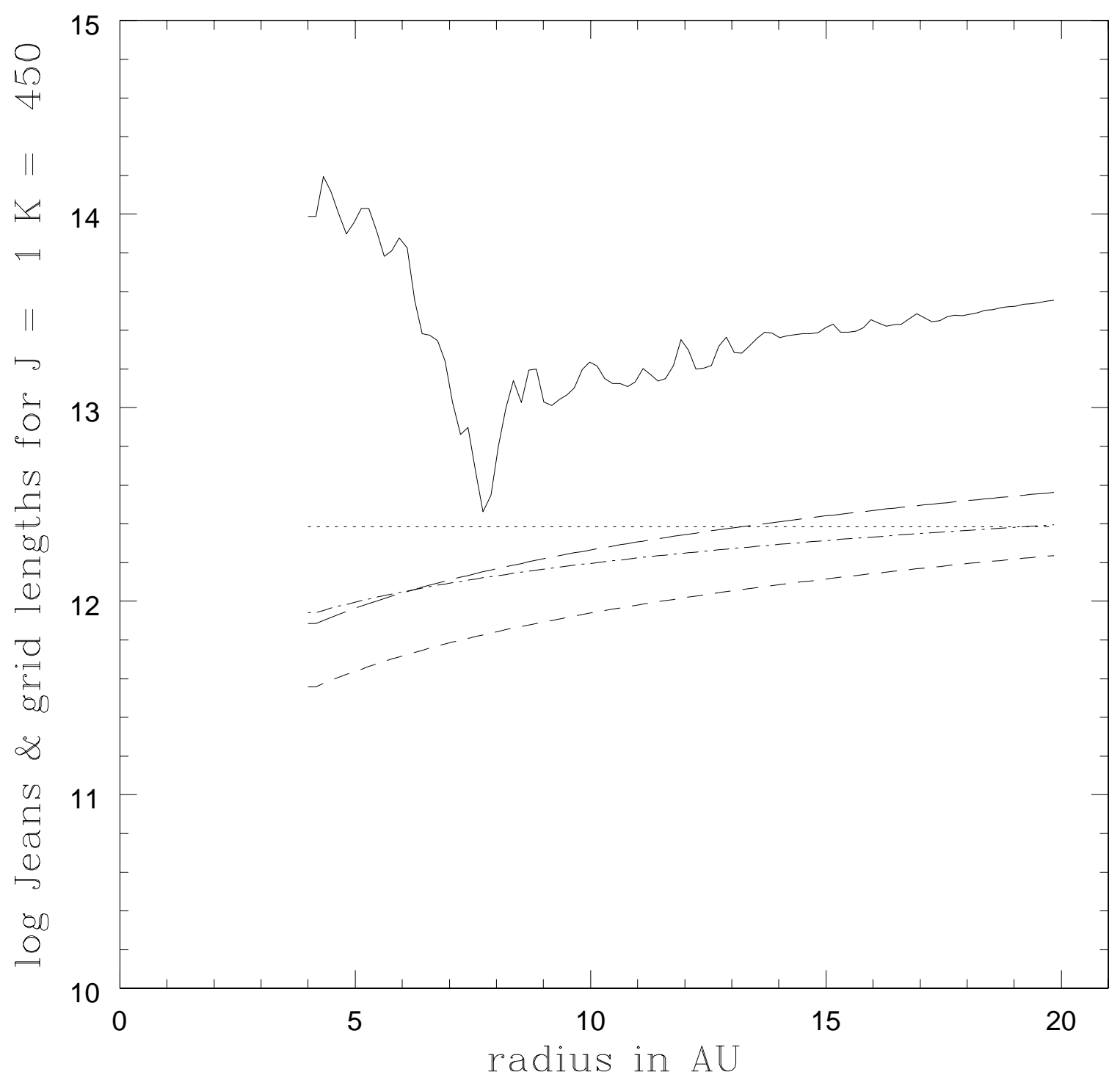

Fig. 6.- Critical Jeans length criterion (solid line) compared to the four spherical coordinate grid spacings $\left(\Delta x_{r}=\Delta r=\right.$ dotted, $\Delta x_{\theta}=r \Delta \theta=$ dashed, $\Delta x_{\phi}=r \sin \theta \Delta \phi=$ long-dashed, and $\Delta x=$ dot-dashed) for model $5 \mathrm{CH}$ after 208 yrs. The radial profile denotes the values for a ray that passes through the density maximum seen at 9 o'clock in Figure 2, leading to the dip in the Jeans criterion at $\sim 8 \mathrm{AU}$. 


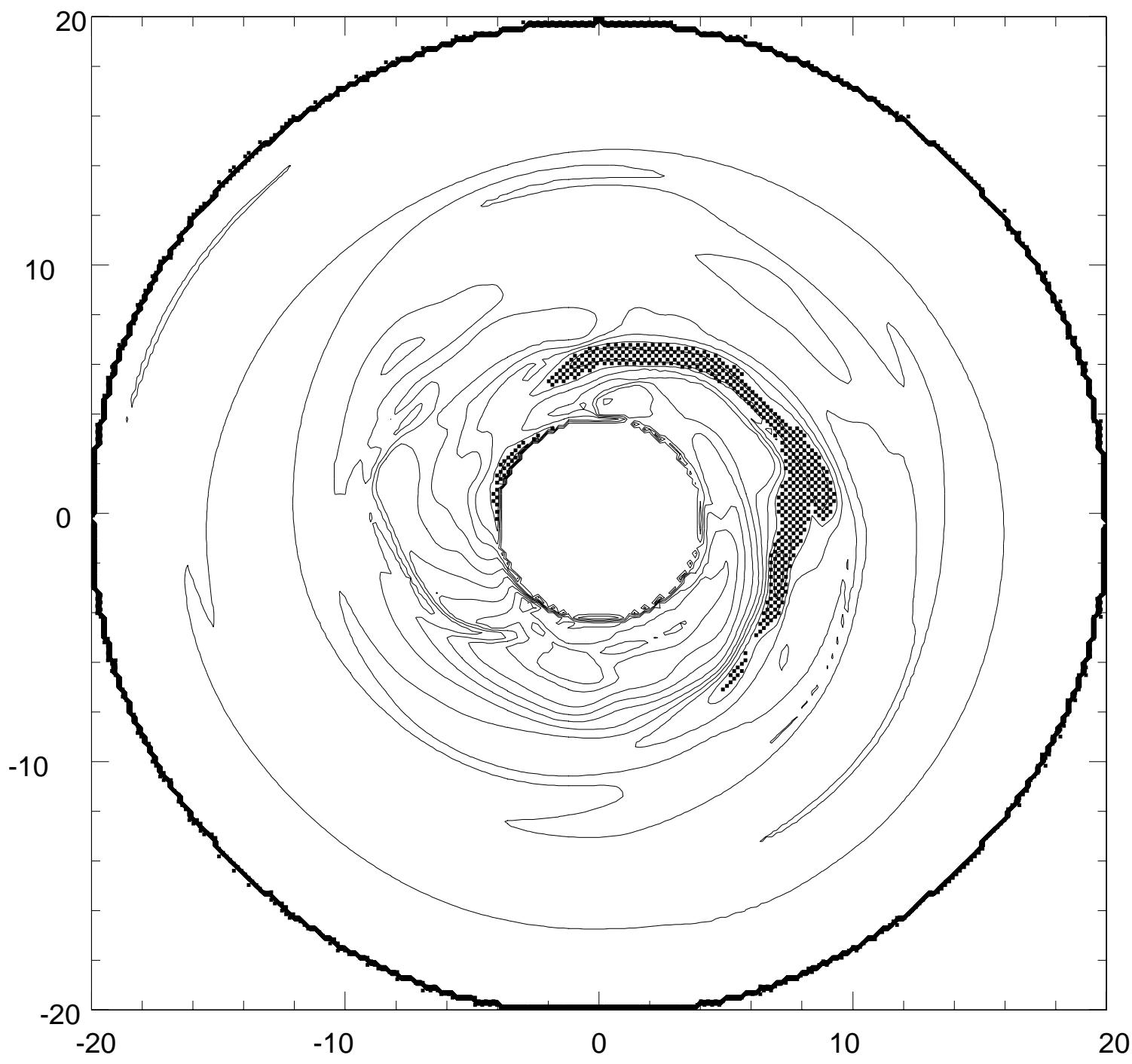

Fig. 7.- Equatorial density contours for model $1 \mathrm{C}$ after 446 yrs of evolution, as in Fig. 2. In a Fourier decomposition of the midplane density in this model, the $m=1$ mode dominates with a maximum amplitude $a_{m=1} \approx 1.5$ at $\sim 8 \mathrm{AU}$, where $a_{m=2} \approx 0.8$ and $a_{m=3} \approx 0.2$. 


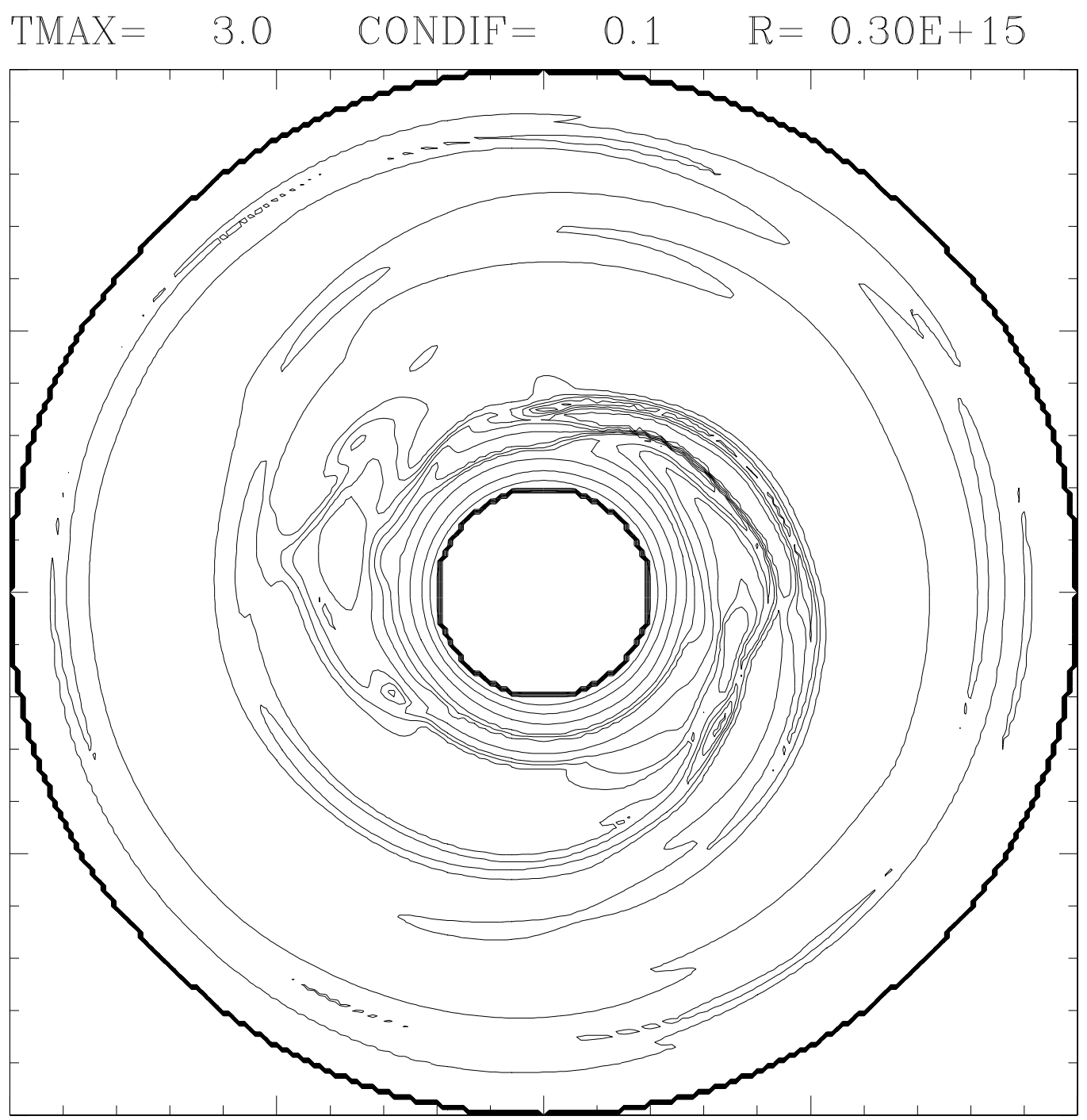

Fig. 8.- Equatorial temperature contours for model $1 \mathrm{C}$ after 446 yrs of evolution, as in Fig. 7 , except that contours represent factors of 1.3 change in temperature. 
Table 1. Initial conditions for the models.

\begin{tabular}{ccccccccc}
\hline \hline model & $M_{s} / M_{\odot}$ & $M_{d} / M_{\odot}$ & $\rho_{o 4}\left(\mathrm{~g} \mathrm{~cm}^{-3}\right)$ & $T_{o}(\mathrm{~K})$ & $T_{e}(\mathrm{~K})$ & $\min \left(Q_{i}\right)$ & $N_{\phi}$ & $N_{Y l m}$ \\
\hline 1A & 0.1 & 0.031 & $1.9 \times 10^{-11}$ & $50 \mathrm{~K}$ & $50 \mathrm{~K}$ & 1.44 & 256 & 32 \\
$1 \mathrm{~B}$ & 0.1 & 0.031 & $1.9 \times 10^{-11}$ & $60 \mathrm{~K}$ & $50 \mathrm{~K}$ & 1.60 & 256 & 32 \\
$1 \mathrm{C}$ & 0.1 & 0.021 & $1.3 \times 10^{-11}$ & $30 \mathrm{~K}$ & $35 \mathrm{~K}$ & 1.53 & 256 & 32 \\
1D & 0.1 & 0.021 & $1.3 \times 10^{-11}$ & $35 \mathrm{~K}$ & $35 \mathrm{~K}$ & 1.65 & 256 & 32 \\
$5 \mathrm{~A}$ & 0.5 & 0.041 & $3.9 \times 10^{-11}$ & $20 \mathrm{~K}$ & $30 \mathrm{~K}$ & 1.40 & 256 & 32 \\
$5 \mathrm{AH}$ & 0.5 & 0.041 & $3.9 \times 10^{-11}$ & $20 \mathrm{~K}$ & $30 \mathrm{~K}$ & 1.40 & 512 & 48 \\
$5 \mathrm{~B}$ & 0.5 & 0.041 & $3.9 \times 10^{-11}$ & $25 \mathrm{~K}$ & $30 \mathrm{~K}$ & 1.56 & 256 & 32 \\
$5 \mathrm{BH}$ & 0.5 & 0.041 & $3.9 \times 10^{-11}$ & $25 \mathrm{~K}$ & $30 \mathrm{~K}$ & 1.56 & 512 & 48 \\
$5 \mathrm{C}$ & 0.5 & 0.065 & $6.0 \times 10^{-11}$ & $50 \mathrm{~K}$ & $50 \mathrm{~K}$ & 1.50 & 256 & 32 \\
$5 \mathrm{CH}$ & 0.5 & 0.065 & $6.0 \times 10^{-11}$ & $50 \mathrm{~K}$ & $50 \mathrm{~K}$ & 1.50 & 512 & 48 \\
$5 \mathrm{D}$ & 0.5 & 0.065 & $6.0 \times 10^{-11}$ & $60 \mathrm{~K}$ & $50 \mathrm{~K}$ & 1.63 & 256 & 32 \\
\hline
\end{tabular}

